\title{
Wall Thickness-Based Adjustment of Ablation Index Improves Efficacy of Pulmonary Vein Isolation in Atrial Fibrillation: Real-Time Assessment by Intracardiac Echocardiography
}

\author{
Yuji Motoike ${ }^{1}$, Masahide Harada ${ }^{1}$, Takehiro Ito ${ }^{1}$, Yoshihiro Nomura ${ }^{1}$, Asuka Nishimura ${ }^{1}$, \\ Masayuki Koshikawa ${ }^{1}$, Eiichi Watanabe ${ }^{2}$, Yukio Ozaki ${ }^{2}$, and Hideo Izawa ${ }^{1}$ \\ ${ }^{1}$ Fujita Health University \\ ${ }^{2}$ Fujita Health University School of Medicine
}

November 2, 2020

\begin{abstract}
Background: Ablation index (AI) linearly correlates with lesion depth and may yield better therapeutic performance in pulmonary vein isolation (PVI) when tailored to a patient's wall thickness (WT) in the left atrium (LA). Methods and results: (First study) In paroxysmal atrial fibrillation patients (PAF, $\mathrm{n}=20)$, the average LA WT $(\mathrm{mm})$ in each anatomical segment for PVI was measured by intra-cardiac echocardiography (ICE) placed in the LA; the optimal AI for creating one-millimeter transmural lesion $(\mathrm{AI} / \mathrm{mm})$ was calculated. (Second study) PAF $(\mathrm{n}=80)$ patients were randomly assigned either to a force-time integral protocol (FTI, 400 gram-second, n=40) or a tailored-AI protocol (TAI, n=40). In TAI, the LA WT in each segment was individually measured by ICE before starting ablation; a target AI was adjusted according to the individual WT in each segment $(\mathrm{AI} / \mathrm{mm} \times \mathrm{WT})$. The acute procedure outcomes and the 1-year AF recurrence rate were compared between FTI and TAI. TAI had higher success rate of first-pass isolation and had lower incidence of residual PV-potentials/conduction gaps after a circular ablation than FTI ( $88 \%$ vs. $65 \%, 15$ vs. $45 \%$, respectively). The procedure time to complete PVI decreased in TAI compared to FTI (52 vs. 83 minutes), being attributed to the increased radiofrequency power and the decreased radiofrequency application time in each point in TAI. TAI had lower 1-year AF recurrence rate than FTI. Conclusion: WT-based AI-adjustment increased acute procedure success, decreased time for PVI, and reduced 1-year AF recurrence rate. Understanding the precise ablation target would improve the efficacy of PVI.
\end{abstract}

\section{Introduction}

Pulmonary vein isolation (PVI) has become the practical standard for catheter ablation of atrial fibrillation $(\mathrm{AF})$. Transmural ablation lesion is crucial for durable PVI. Insufficient radiofrequency (RF) current delivery may result in incomplete lesion formation and thus require repeat procedures; in contrast, excessive RF current may contribute to serious complications such as steam pop formation, cardiac tamponade, and esophageal injury.

Previous histological studies demonstrated that the left-atrium (LA) and the area surrounding the pulmonary vein (PV) are anatomically heterogenous. Wall thickness (WT) also varies among patients. ${ }^{1,2}$ Understanding the precise ablation target in each patient will improve the ablation procedure and reduce the risk of adverse events.

Ablation index $(\mathrm{AI})$ is a marker incorporating contact force $(\mathrm{CF})$, time, and power. It is linearly correlated with lesion depth. ${ }^{3}$ Therefore, the adjustment of AI according to the WT in the area surrounding the PV in each patient seems to yield better therapeutic performance in PVI. Intracardiac echocardiography (ICE) is utilized to visualize the cardiac anatomy during the ablation procedure. We assumed that the WT in the 
area surrounding the PV could be measured by ICE in a real-time manner and the AI-adjustment based on each patient's WT would improve the procedure of PVI.

The aims of this study are to measure the WT in the area surrounding the PV using ICE and to assess whether or not WT-based AI-adjustment improves the procedure of PVI.

\section{Methods}

\section{Patients}

This study protocol consisted of two parts: the first study measured average LA WT ( $\mathrm{mm})$ in each anatomical segment for PVI by intra-cardiac echocardiography (ICE) and calculated optimal AI for creating 1-mm transmural lesion $(\mathrm{AI} / \mathrm{mm})$. The second study was a prospective randomized study to compare the procedure characteristics of PVI between a force-time integral protocol and a tailored-AI protocol, following the first study (Figure 1).

From May 2018 to September 2019, 100 paroxysmal AF patients undergoing PVI in Fujita Health University were eligible. The review board of Fujita Health University School of Medicine approved the protocol and all patients gave written informed consent. Baseline demographics and clinical information were obtained, and laboratory examinations were performed before the PVI. Left-atrial diameter (LAD), left-ventricular systolic/diastolic dimensions, and left-ventricular ejection fraction (LVEF) were assessed by transthoracic echocardiography. A 3-dimensional image of the LA/PV geometry was reconstructed by cardiac computed tomography $(\mathrm{CT})$ imaging.

All patients received oral anticoagulation therapy with vitamin $\mathrm{K}$ antagonist (VKA) or non-vitamin $\mathrm{K}$ antagonist oral anticoagulant (NOAC) for [?]4 weeks prior to the catheter ablation. In patients taking VKA, an international normalized ratio was controlled between 2.0 and 3.0. VKA and NOAC were used without interruption during the procedure. Transesophageal echocardiography was performed one day before catheter ablation to detect LA thrombus.

Exclusion criteria were as follows: patients aged under 18 years old, previous ablation procedure for AF, prior prosthetic mitral valve replacement, severe structural cardiac abnormality (e.g., congenital heart disease), severe left ventricular systolic dysfunction $(\mathrm{LVEF}<35 \%$ ), severe renal dysfunction (creatinine clearance $<15$ $\mathrm{ml} / \mathrm{min}$ )/hemodialysis, LA thrombus detected by TEE, and pregnancy.

All anti-arrhythmic drugs were stopped 5 days prior to the procedure. No patient was being treated with amiodarone.

\section{Procedure}

In all patients, the procedure was performed under deep sedation with a continuous intravenous infusion of dexmedetomidine hydrochloride (Precedex) and additional boluses of midazolam and thiamylal. Esophageal temperature was monitored throughout the procedure; temperature limit was set to 41 . If a patient showed AF rhythm in the electrophysiological laboratory, electric cardioversion was performed to restore sinus rhythm.

A bolus of 5,000-10,000 international units of unfractionated heparin (100-150 U/kg) was administered before trans-septal puncture to achieve activated clotting time $(\mathrm{ACT})>300 \mathrm{sec}$. ACT was measured every $20 \mathrm{~min}$ after the first heparin shot and additional heparin boluses were administered to maintain the ACT $>300$ sec. A decapolar catheter was advanced into the coronary sinus via the internal jugular vein. An 8 Fr ICE catheter (SoundStar, Biosense Webster, Diamond Bar, CA, USA) was inserted into the right atrium (RA) via a 10Fr short sheath in the right femoral vein; trans-septal puncture was performed under ICE guidance. Two 8Fr long sheaths (SL1; Abbott, St. Paul, MN, USA) and a 8.5Fr deflectable sheath (Agilis NxT, [short size $61 \mathrm{~cm}$ ], Abbott, St. Paul, MN, USA) were then advanced into the LA. A ring-shaped decapolar catheter (LASSOß) eco catheter, Biosense Webster, Diamond Bar, CA, USA) and a multielectrode catheter (PENTARAY (R) eco catheter, Biosense Webster) were used for mapping. CARTO V6 (Biosense Webster) was used to create a 3-dimensional electro-anatomical voltage map of the LA and to integrate the voltage map with the CT 
imaging reconstruction of the LA (CartoMerge, Biosense Webster). The contrast fluoroscopy image of the LA was obtained and combined with the reconstructed CT image by CARTOUNIVU Module (Biosense Webster).

\section{ICE and WT measurement}

ICE imaging in the LA was performed before starting PVI. The ICE catheter, equipped with a linearphased array multifrequency (5.5-10 MHz) transducer, was connected to an Acuson P500 system (SIEMENS, Munich, Germany). The ICE catheter in the RA was withdrawn after the trans-septal puncture and then advanced into the LA via the $8.5 \mathrm{Fr}$ deflectable sheath (Agilis NxT, short size). Only the tip (transducer) of ICE catheter was carefully put out from the deflectable sheath and was placed in the center of the LA under fluoroscopic guidance: the safe distance (more than $10 \mathrm{~mm}$ ) between the tip and the LA endocardium was always confirmed and maintained throughout manipulation of the ICE catheter (Figure 2). The ICE catheter was finely rotated without any deflection (keeping neutral position) around the PV-junctional area to obtain images perpendicular to the LA/PV surface. When different angulation of ultrasound beam was required for clear anatomical visualization, only the deflectable sheath was manipulated and deflected to change the position of the ICE transducer and the direction of the ultrasound beam. The snapshots of ICE imaging in each segment for PVI were obtained during the diastolic phase in eight segments: the anterior, roof, posterior and bottom of both PVs. The endocardial boundary (separating the blood pool from myocardium) and the epicardial boundary (separating the myocardial tissue from other surrounding tissue) were manually outlined. The distance between the epicardial and endocardial boundary was measured and defined as the WT. The WT was measured more than five times in each segment between $5 \mathrm{~mm}$ inside and $5 \mathrm{~mm}$ outside of the LA-PV junction; the highest and lowest measurements were discarded and the average of the [?]3 middle values was considered as the $\mathrm{WT}$ in a segment.

To validate the accuracy and reliability of the ICE-based WT measurements, the LA WTs on cardiac CT were also measured in the same patient. The cardiac CT-based measurement was performed by cardiologists who were blinded to the result of the ICE-based measurement; the WT values were compared between the ICE- and cardiac CT-based measurements.

\section{PVI}

PVI was performed by two electrophysiologists (MH and MK) with more than 500 case experiences of AF ablation before the study. RF energy was applied in a point-by-point manner using an open-irrigated catheter

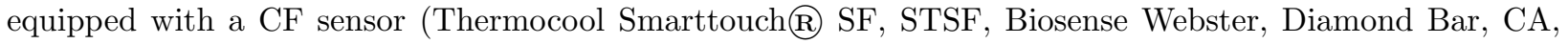
USA).

Before AI technology was introduced to our facility, AI-blinded retrospective analysis was performed by the medical device manufacturer (Biosense Webster) to estimate optimal AI for a first-pass isolation in standard $\mathrm{PVI}(\mathrm{n}=5$ in each operator).

Target CF was10-30g (irrigation flow rate: $8 \mathrm{ml} / \mathrm{min}$ for [?]30W, $15 \mathrm{ml} / \mathrm{min}$ for $>30 \mathrm{~W}$, power control mode). Contiguous RF region was ensured by targeting an inter-lesion distance [?]6 $\mathrm{mm} .{ }^{4}$ The ablation lesion sets encircling the PV antra were created $>5 \mathrm{~mm}$ outside the PV ostia, where the local electrograms did not show near-field PV signals. For the anterior segment in the left-PV (the left lateral ridge), RF delivery was applied only to the inside $(<5 \mathrm{~mm})$ of the left-PV ostium. The RF ablation on the inter-PV carina was not performed as a part of initial lesion set, unless it was needed to achieve PVI. No additional linear ablation in the LA was performed, and only cavotricuspid isthmus ablation was permitted for documented typical atrial flutter. RF energy was delivered at 30-40W in power-controlled mode but was decreased to $20-30 \mathrm{~W}$ at the posterior/bottom segments if esophageal temperature exceeded 39 (irrigation flow rate: $8 \mathrm{ml} / \mathrm{min}$ for [?] $30 \mathrm{~W}, 15 \mathrm{ml} / \mathrm{min}$ for $>30 \mathrm{~W}$ ). The LASSO NAV catheter was placed inside the ablation line of PVI. The success of first-pass isolation was defined as the electrical isolation of ipsilateral PV which occurred either before or at completion of the RF lesion sets without the ablation of the inter-PV carina. After the circular

$\mathrm{RF}$ application, electro-anatomical mapping was performed using the PENTARAY catheter. If residual PV potentials and/or conduction gaps within the isolated area were observed, additional RF ablation was 
performed to eradicate them. Conduction block from the PV to the LA was confirmed by high-output pacing (20 V output, 1 msec pulse) from the isolated PV area at the both sides. After a 20-minute waiting period, boluses of isoproterenol $(2-4 \mu \mathrm{g})$ and adenosine triphosphate $(20 \mathrm{mg})$ was administered. If spontaneous PV reconnection and/or drug-evoked dormant conduction were observed, an additional RF application was done to eliminate it.

\section{Ablation Protocols}

In the second study, PAF patients were randomly assigned either to a force-time integral-based (FTI) protocol or a tailored-AI-based (TAI) protocol.

(1) FTI protocol: Target FTI was set as [?] 400 gram·second [ $\mathrm{g} \cdot \mathrm{s}]$ regardless of RF power and WT according to the previous EFFICAS-I based CF guidelines. ${ }^{5}$

(2) TAI protocol: WT in each segment for PVI was measured in individuals before starting ablation; a target AI was individually adjusted according to the WT in each anatomical segment.

Eighty patients were randomly assigned either to FTI $(n=40)$ or to TAI $(n=40)$. When a target FTI/AI could not be reached because of catheter displacement, RF was recommenced at the same point until the target FTI/AI was achieved. If RF delivery had to be terminated due to a rise in esophageal temperature or an abrupt impedance drop ( $>20 \mathrm{ohm})$, a subtherapeutic FTI/AI lesion was accepted. The upper and the lower limits of target AI were set to 600 and 300, respectively.

The characteristics of the procedure, including the percentage of first-pass isolation, the percentage of residual PV potentials and/or conduction gap, the incidence of the spontaneous PV reconnection and/or drug-evoked dormant conduction, and the procedure time to complete PVI, were compared between FTI and TAI.

\section{Ablation Lesion Data Analysis}

The STSF catheter was used for acquisitions of FTI/AI and ablation lesion data. The calibration of the STSF catheter was performed every 20 minutes. The settings of the automatic lesion annotation software (VISITAG $^{\mathrm{TM}}$ Module, Biosense Webster) were as follows: for location stability, minimum time was 3 seconds and maximum range $3.0 \mathrm{~mm}$; force over time was $25 \%$ with a minimum force of $3 \mathrm{~g}$. The lesion tag size was a $2 \mathrm{~mm}$ radius. The parameters for each VISITAG ${ }^{\mathrm{TM}}$ ablation point were as follows: RF application time, average $\mathrm{CF}, \mathrm{RF}$ power, impedance drop (the difference between the pre-ablation and the lowest recorded impedance values during ablation), FTI, and AI. The data were compared between FTI and TAI protocols.

\section{Follow-up}

All patients were followed up by cardiologists at the outpatient department in Fujita Health University at $1,3,6,9$, and 12 months after ablation. All patients were asked about their symptoms and underwent a 12-lead electrocardiogram. Holter ECG monitoring was performed at 6 and 12 months follow-up. In case of AF recurrence within a 3-month blanking period, an anti-arrhythmic drug was prescribed and discontinued after the blanking period. AF recurrence was defined as any atrial tachy-arrhythmias lasting more than 30 seconds and occurring after the blanking period. The AF-free survival rates at more than six months were compared between FTI and TAI protocols.

\section{Statistical Analysis}

Continuous variables, represented as mean \pm standard deviation, were compared using unpaired t-tests. Categorical data, expressed as frequencies and percentages, were compared using chi-square tests. The followup period was calculated from the date of registration to that of the AF recurrence or censoring. AF-free survival rate was calculated using Kaplan-Meier survival analysis, and log-rank statistics were used for group comparisons.

All tests were 2 -sided, and a p value $<0.05$ was considered statistically significant. Statistical analyses were performed using JMP11 (SAS Institute, Cary, NC, USA). 


\section{Results}

\section{The First Study}

WTs were evaluated by ICE in 20 patients. Baseline characteristics in patients for the first ICE study are shown in Supplemental Table 1. Figure 3 shows representative ICE images and WT measurements. The LA WTs differed between segments. Table 1 summarizes the measurements of LA WTs. The anterior segment of the left-PV (the left lateral ridge, LLR) had the thickest average WT $(6.0 \pm 1.3 \mathrm{~mm})$ among all segments. The morphology of LAA varies among patients (Figure 4).

To validate the accuracy and reliability of our ICE-based WT measurement, we also measured WTs using cardiac CT in the same patients $(\mathrm{n}=6)$. The ICE-based WTs measurement were almost similar to, or a little bit thicker than, the cardiac CT-based WTs measurement (Supplemental Figure 1 and Supplemental Table 2).

\section{Procedure Characteristics}

In the second study, PAF patients $(\mathrm{n}=80)$ were randomly assigned either to the FTI protocol (FTI [?]400 $\left.\mathrm{g}^{*} \mathrm{~s}, \mathrm{n}=40\right)$ or the TAI protocol $(\mathrm{n}=40)$. Our AI-blinded retrospective analysis demonstrated that AI 530 was enough for a success of first-pass isolation and was supposed to create transmural lesion in the thickest WT segment and in the first study; the left-PV (the LLR) had the thickest average WT. Optimal AI for creating transmural lesion per millimeter $(\mathrm{AI} / \mathrm{mm})$ was therefore calculated by AI 530 divided by $6.0 \mathrm{~mm}$ (the average $\mathrm{WT}$ in the LLR), resulting in $90 \mathrm{AI} / \mathrm{mm}$. In TAI, target AI in each segment was calculated by the formula as follows: $[90(\mathrm{AI} / \mathrm{mm}) \mathrm{x}$ individual $\mathrm{WT}(\mathrm{mm})$ in each segment].

Table 2 shows the baseline characteristics in FTI and TAI; there were no significant differences in the patient demographics between the both groups. In TAI, the average WT and target AI in each segment are shown in Figure 5, and these values in each patient are shown in Supplemental Table 3. PVI was completed in all patients.

The success rate of first-pass isolation was significantly higher in TAI than in FTI $(88 \%$ and $65 \%$, Figure $6 \mathrm{~A})$. The prevalence of residual conduction gap/PV potential after a circular RF application was significantly lower in TAI than in FTI (15\% and 45\%, Figure $6 \mathrm{~B})$. The incidence of spontaneous PV reconnection/drugevoked dormant conduction were comparable between the two groups (18\% and $21 \%$, Figure 6C). The mean procedure time to complete PVI significantly decreased in TAI than in FTI (52+-17 min and 83+-27

min, p $<0.05$, Figure 6D), showing $37 \%$ reduction by TAI. These suggest that FAI improves the efficacy of PVI procedure by increasing the rate of first-pass isolation with reducing the residual conduction gap/PV potentials, resulting in shortening the time for PVI.

\section{VISITAG Data}

A total of 1316 ablation points in 20 patients ( $\mathrm{n}=10$ for FTI, $\mathrm{n}=10$ for TAI, Table 3 ) were analyzed. The number of RF application of points (VisiTag) were unchanged between FTI and TAI in each PV (Table 3). There was no significant difference in CFs between FTI and TAI in all segments except for RPV posterior and bottom. FTI values in most anatomical segments decreased in TAI versus FTI whereas RF power increased in TAI. The impedance drop was also comparable between FAI and TAI in all regions, except for the LPV anterior segment. RF application time per lesion significantly decreased in TAI compared to FTI. These suggest that AI-monitored RF application safely allows higher power and shorter duration of RF energy application per VisiTag point (Table 3).

\section{Complication and Follow-up}

No procedure-related complications occurred in either group during or after the procedure. Thirteen patients had AF recurrence after the 3-month blanking period ( $\mathrm{n}=11$ in FTI, $\mathrm{n}=2$ in TAI). TAI showed a higher 1-year AF-free survival rate than FTI (Figure 7).

\section{Discussion}


The major findings of this study are as follows. WTs in the area surrounding the PV, evaluated by ICE, were heterogenous and varied among patients. In addition, real-time WT measurement and WT-based AIadjustment in each patient increased success rate of first-pass isolation, decreased residual gap/PV potentials after a circular RF application, and decreased the time to complete PVI. Finally, TAI showed a higher 1-year AF-free survival rate than FTI. We first conducted real-time ICE measurement of the LA WT for PVI and then performed WT-based AI adjustment in each anatomical segment. Effective use of AI technology based on the precise RF target could improve the efficacy of PVI.

\section{Comparison with Previous Studies}

LA WTs have been evaluated in post-mortem studies, revealing a significant variation in the WTs among regions, patients, and studies. ${ }^{1,2}$ Most of these studies reported average thickness of LA myocardium between 1.0 and $5.0 \mathrm{~mm}$ with a range from 0.5 to $12 \mathrm{~mm} .{ }^{1,2}$ The results in this study were almost within this range but the average WT values were slightly thicker than those in previous post-mortem studies. We measured the distance between endocardial and epicardial boundary, which might be greater than the actual muscular thickness in histological examinations. Fresh tissue specimens also shrink after formalin fixation, causing a reduction of WT. These factors likely contribute to the difference in the WT measurement between previous histological studies and our ICE study. Cardiac CT imaging has been used to evaluate LA WT in $\mathrm{AF}$ patients, ${ }^{2,6-8}$ and therefore we compared the ICE-based WTs measurements to cardiac CT-based WT measurements in the same patients to validate the accuracy and reliability of our method; the ICE-based WT measurements were almost similar to, or slightly thicker than, the cardiac CT-based WT measurements in our study, suggesting the similar accuracy of these measurements. The CT-derived WT averages approximately 1.0-3.0 $\mathrm{mm}$ in previous reports. Of note, when viewed alongside the results of the pathological studies in the similar location, the CT measurements are reportedly lower than the histological measurement. ${ }^{2}$

The left-PV anterior segment, the LLR, had the thickest wall among all segments in this study. Cabrera et al. have reported that this region has a folding structure with the thick muscle ranging from $2.2 \mathrm{~mm}$ to 12.3 mm. ${ }^{9}$ The morphology can be flat, round, or pointed, as seen in this study (Figure 4). ${ }^{9}$ A previous CT study has also shown that the LLR had the thickest walls in the area surrounding the PV, which is consistent with our results. ${ }^{6}$

ICE provides higher spatial resolution $(0.2-0.3 \mathrm{~mm})$ than cardiac CT $(0.4 \mathrm{~mm}-)$ and MRI $(1.4 \mathrm{~mm}-)^{2,10}$ and therefore seems to be a good modality for the WT evaluation in the LA. ICE was used to assess right atrial WTs in an experimental study. ${ }^{11}$ However, it has not been used to assess WT in the LA of AF patients, likely because of the invasive nature of this examination. The ICE has been limited to use in procedures where its value has been clearly established. The ICE catheter is usually placed in the RA for the trans-septal puncture during AF ablation. In this study, the ICE catheter was advanced into the LA, allowing clearer visualization of the anatomy of the area surrounding the PV and reducing the angulation errors of the view perpendicular to the LA/PV surface for the WT measurement. Nevertheless, this assessment lacks a gold standard and merits further evaluation.

\section{Customized AI Approach}

Various target AI values have been proposed in previous studies, being rounded up from 400 to 550 for the anterior/roof segments and from 330 to 400 for the posterior/bottom segments. The AI-guided PVI produced the higher acute procedure success and the lower AF recurrence rate. ${ }^{4,12-15}$ However, target AIs in the previous studies were usually based on a physician's expertise and experience, not on a precise ablation target.

WT in the area surrounding the PV is heterogenous and varies among patients; a single AI cut-off approach might not be suitable to all patients. Haines et al. designed an open-irrigated RF ablation catheter with an ultrasound transducer at the tip. They performed near-field ultrasound imaging with this catheter in animal experiments. This approach could assess WT and lesion depth of ablation required for transmural lesion formation to optimize power delivery. ${ }^{16} \mathrm{AI}$ was correlated with lesion depth, width, and volume in an experimental study; ${ }^{17}$ it seems to yield better therapeutic performance when adjusted to the precise ablation 
target. WT-based AI-adjustment in this study would exploit the unique advantage of AI-guided ablation to its full potential. To date, the RF delivery method has been extensively discussed for durable PVI, but the precise ablation target also deserves more attention. Nevertheless, we had a few cases of first-pass isolation failure, the acute reconnection, and AF recurrence, suggesting that our protocol should be further optimized.

It has recently been reported that high power-short duration (HPSD) ablation decreased the total RF application duration of PVI in paroxysmal AF patients. ${ }^{18}$ Experimental studies demonstrated that HPSD $(90 \mathrm{~W}, 4 \mathrm{sec})$ creates wider but shallower lesions than conventional RF ablation, ${ }^{19}$ implying that the WT in a target RF region is important for effective HSPD ablation. The HSPD ablation may be inadequate for a thicker WT area and the precise WT evaluation may help to determine the most suitable region for the HSPD ablation.

\section{Limitation}

This is a single-center study of a small number of patients. Although ECG and Holter monitoring was regularly performed during the 1-year follow-up, AF recurrence may be underestimated. The ICE catheter was placed in the LA to obtain a clear image of the area surrounding the PV. Nevertheless, this may still cause angulation errors in the direction of the ultrasound beam perpendicular to the LA/PV surface and distort the measurement.

\section{Conclusion}

WT in the area surrounding the PV varies among patients. WT-based AI-adjustment increased acute procedure success, decreased time for PVI, and reduced 1-year AF recurrence rate. Understanding the precise ablation target would therefore improve the efficacy of PVI.

\section{Acknowledgement}

This research did not receive any specific grant from funding agencies in the public, commercial, or not-forprofit sectors.

\section{References}

1. Ho SY, Cabrera JA, Sanchez-Quintana D. Left atrial anatomy revisited.

Circ Arrhythm Electrophysiol. 2012;5:220-228.

2. Whitaker J, Rajani R, Chubb H, Gabrawi M, Varela M, Wright M, Niederer S, O'Neill MD. The role of myocardial wall thickness in atrial arrhythmogenesis. Europace. 2016;18:1758-1772.

3. Nakagawa H, Ikeda A, Govari A, Papaioannou T, Constantine G, Bar-Tal M. Prospective study using a new formula incorporating contact force, radiofrequency power and application time (Force-Power-Time Index) for quantifying lesion formation to guide long continuous atrial lesions in the beating canine heart. Circulation. 2013; 128: A12104.

4. Phlips T, Taghji P, El Haddad M, Wolf M, Knecht S, Vandekerckhove Y, Tavernier R, Duytschaever M. Improving procedural and one-year outcome after contact force-guided pulmonary vein isolation: the role of interlesion distance, ablation index and contact force variability in the 'CLOSE'-protocol. Europace. 2018;20:f419-f427.

5. Neuzil P, Reddy VY, Kautzner J, Petru J, Wichterle D, Shah D, Lambert H, Yulzari A, Wissner E, Kuck KH. Electrical reconnection after pulmonary vein isolation is contingent on contact force during initial treatment: results from the EFFICAS I study. Circ Arrhythm Electrophysiol. 2013;6:327-333.

6. Beinart R, Abbara S, Blum A, Ferencik M, Heist K, Ruskin J, Mansour M. Left atrial wall thickness variability measured by $\mathrm{CT}$ scans in patients undergoing pulmonary vein isolation.

J Cardiovasc Electrophysiol. 2011;22:1232-1236. 
7. Takahashi K, Okumura Y, Watanabe I, Nagashima K, Sonoda K, Sasaki N, Kogawa R, Iso K, Ohkubo K, Nakai T, Hirayama A. Relation Between Left Atrial Wall Thickness in Patients with Atrial Fibrillation and Intracardiac Electrogram Characteristics and ATP-Provoked Dormant Pulmonary Vein Conduction. J Cardiovasc Electrophysiol. 2015;26:597-605.

8. Suenari K, Nakano Y, Hirai Y, Ogi H, Oda N, Makita Y, Ueda S, Kajihara K, Tokuyama T, Motoda C, Fujiwara M, Chayama K, Kihara Y. Left atrial thickness under the catheter ablation lines in patients with paroxysmal atrial fibrillation: insights from 64-slice multidetector computed tomography.

Heart Vessels. 2013;28:360-368.

9. Cabrera JA, Ho SY, Climent V, Sanchez-Quintana D. The architecture of the left lateral atrial wall: a particular anatomic region with implications for ablation of atrial fibrillation.

Eur Heart J. 2008;29:356-362.

10. Karim R, Blake LE, Inoue J, Tao Q, Jia S, Housden RJ, Bhagirath P, Duval JL, Varela M, Behar JM, Cadour L, van der Geest RJ, Cochet H, Drangova M, Sermesant M, Razavi R, Aslanidi O, Rajani R, Rhode K. Algorithms for left atrial wall segmentation and thickness - Evaluation on an open-source CT and MRI image database. Med Image Anal. 2018;50:36-53.

11. Ren JF, Callans DJ, Schwartzman D, Michele JJ, Marchlinski FE. Changes in local wall thickness correlate with pathologic lesion size following radiofrequency catheter ablation: an intracardiac echocardiographic imaging study. Echocardiography. 2001;18:503-507.

12. Das M, Loveday JJ, Wynn GJ, Gomes S, Saeed Y, Bonnett LJ, Waktare JEP, Todd DM, Hall MCS, Snowdon RL, Modi S, Gupta D. Ablation index, a novel marker of ablation lesion quality: prediction of pulmonary vein reconnection at repeat electrophysiology study and regional differences in target values. Europace. 2017;19:775-783.

13. Dhillon G, Ahsan S, Honarbakhsh S, Lim W, Baca M, Graham A, Srinivasan N, Sawhney V, Sporton S, Schilling RJ, Chow A, Ginks M, Sohal M, Gallagher MM, Hunter RJ. A multicentered evaluation of ablation at higher power guided by ablation index: Establishing ablation targets for pulmonary vein isolation. J Cardiovasc Electrophysiol. 2019;30:357-365.

14. Hussein A, Das M, Riva S, Morgan M, Ronayne C, Sahni A, Shaw M, Todd D, Hall M, Modi S, Natale A, Dello Russo A, Snowdon R, Gupta D. Use of Ablation Index-Guided Ablation Results in High Rates of Durable Pulmonary Vein Isolation and Freedom From Arrhythmia in Persistent Atrial Fibrillation Patients. Circ Arrhythm Electrophysiol. 2018;11:e006576.

15. Solimene F, Schillaci V, Shopova G, Urraro F, Arestia A, Iuliano A, Maresca F, Agresta A, La Rocca V, De Simone A, Stabile G. Safety and efficacy of atrial fibrillation ablation guided by Ablation Index module. J Interv Card Electrophysiol. 2019;54:9-15.

16. Haines DE, Wright M, Harks E, Deladi S, Fokkenrood S, Brink R, Belt H, Kolen AF, Mihajlovic N, Zuo F, Rankin D, Stoffregen W, Cockayne D, Cefalu J. Near-Field Ultrasound Imaging During Radiofrequency Catheter Ablation: Tissue Thickness and Epicardial Wall Visualization and Assessment of Radiofrequency Ablation Lesion Formation and Depth.

Circ Arrhythm Electrophysiol. 2017;10:e005295.

17. Kawaji T, Hojo S, Kushiyama A, Nakatsuma K, Kaneda K, Kato M, Yokomatsu T, Miki S. Limitations of lesion quality estimated by ablation index: An in vitro study.

J Cardiovasc Electrophysiol. 2019;30:926-933.

18. Reddy VY, Grimaldi M, De Potter T, Vijgen JM, Bulava A, Duytschaever MF, Martinek M, Natale A, Knecht S, Neuzil P, Purerfellner H. Pulmonary Vein Isolation With Very High Power, Short Duration, Temperature-Controlled Lesions: The QDOT-FAST Trial. 
JACC Clin Electrophysiol. 2019;5:778-786.

19. Bourier F, Duchateau J, Vlachos K, Lam A, Martin CA, Takigawa M, Kitamura T, Frontera A, Cheniti G, Pambrun T, Klotz N, Denis A, Derval N, Cochet H, Sacher F, Hocini M, Haissaguerre M, Jais P. Highpower short-duration versus standard radiofrequency ablation: Insights on lesion metrics.

J Cardiovasc Electrophysiol. 2018;29:1570-1575.

\section{Figure Legends}

Figure 1. Schematic diagram of the study protocol.Target AI in FAI was based on our previous retrospective study. Target AI in TAI was calculated in each patient by the formula as follows: target AI in a segment $=90(\mathrm{AI} / \mathrm{mm}) \mathrm{x}$ individual WT $(\mathrm{mm})$ in each segment. AI, ablation index; ICE, intracardiac echocardiography; PV, pulmonary vein; WT, wall thickness.

Figure 2. Biplane fluoroscopic images of ICE manipulation in the LA. (A) Contrast-enhanced fluoroscopic image of the LA. AP view. (B) LAO 60deg view. (C) ICE manipulation under fluoroscopic guidance. AP view. (D) LAO 60deg view. AP, anterior-posterior. ICE, intracardiac echocardiography. LAO, left anterior oblique.

Figure 3. Representative snapshots of ICE imaging. (A) ICE images of the roof (upper left), bottom (upper middle), and posterior (upper right) segments in the left-PV (LPV). The direction of ultrasound beam (left) and the corresponding snapshot of ICE imaging (right) are shown in each segment. Red arrows indicate the measurement of wall thickness (WT). (B) ICE images of the anterior (lower left), roof (lower middle), and bottom (lower right) segments in the right-PV (RPV). Ao, aorta; HRA-CS, high right-atriumcoronary sinus; LA, left-atrium; LPA/RPA, left/right pulmonary artery; LSPV/LIPV, left superior/inferior pulmonary vein; RSPV/RIPV, right superior/inferior pulmonary vein; SVC, superior vena cava.

Figure 4. Representative ICE images of the anterior segment in the left-PV (left lateral ridge, LLR) . The direction of ultrasound beam (left) and the corresponding snapshot of ICE imaging (right) are shown in each patient. Patient \#3 had a left common PV. AV, aortic valve; LAA, left atrial appendage; LSPV/LIPV, left superior/inferior pulmonary vein.

Figure 5. Average WT and target AI in each segment for PVI in TAI. AI, ablation index; LPV, left pulmonary vein; RPV, right pulmonary vein; WT, wall thickness.

Figure 6. Comparison of procedure characteristics between FTI and TAI. (A) Mean+-SD success rate of first-pass isolation. Open column indicates FTI and solid column does TAI. (B) Mean+-SD prevalence of residual PV potential/conduction gap after a circular RF application. (C) Mean+-SD incidence of spontaneous PV reconnection/drug-evoked dormant conduction. (D) Mean+-SD RF ablation time to complete PVI. n.s., not significant. ${ }^{*}$ p $<0.05$ vs. FTI.

Figure 7. Kaplan-Meier AF-free survival curve in FTI and TAI

Table 1. Average Wall Thickness in First ICE Study

\begin{tabular}{ll}
\hline Segment & Wall Thickness, $\mathrm{mm}$ \\
\hline Left-PV & \\
anterior & $6.0 \pm 1.3$ \\
roof & $5.1 \pm 1.5$ \\
posterior & $4.3 \pm 1.4$ \\
bottom & $4.8 \pm 1.1$ \\
Right-PV & \\
anterior & $5.6 \pm 1.4$ \\
roof & $5.2 \pm 2.2$ \\
posterior & $4.3 \pm 1.5$
\end{tabular}




\begin{tabular}{ll}
\hline Segment & Wall Thickness, $\mathrm{mm}$ \\
\hline bottom & $3.8 \pm 0.9$ \\
\hline
\end{tabular}

Table 2. Comparison of Patient Characteristics between FTI and TAI

\begin{tabular}{llll}
\hline & FTI $(\mathrm{n}=40)$ & TAI $(\mathrm{n}=40)$ & p-value \\
\hline Age, y/o & $67.6 \pm 8.5$ & $69.4 \pm 10.8$ & 0.424 \\
Male, n (\%) & $26(65 \%)$ & $22(55 \%)$ & 0.246 \\
BMI, kg/m2 & $24.4 \pm 3.1$ & $24.4 \pm 2.9$ & 0.991 \\
CHADS $_{2}$ score, points & $1.5 \pm 1.2$ & $1.4 \pm 1.4$ & 0.860 \\
CHA $_{2}$ DS $_{2}$-VASc score, points & $2.6 \pm 1.6$ & $2.6 \pm 1.8$ & 0.895 \\
CHF, n (\%) & $12(30 \%)$ & $10(25 \%)$ & 0.441 \\
HT, n (\%) & $25(63 \%)$ & $22(55 \%)$ & 0.651 \\
DM, n (\%) & $6(15 \%)$ & $4(10 \%)$ & 0.368 \\
Stroke/TIA, n (\%) & $3(8 \%)$ & $3(8 \%)$ & 0.662 \\
Vascular Disease, n (\%) & $0(0 \%)$ & $0(0 \%)$ & - \\
Medications, n (\%) & & & \\
ACEI/ARB, n (\%) & $19(48 \%)$ & $11(28 \%)$ & 0.050 \\
$\beta$ blocker, n (\%) & $22(55 \%)$ & $19(48 \%)$ & 0.327 \\
AAD, n (\%) & $7(18 \%)$ & $5(13 \%)$ & 0.398 \\
Laboratory data & & & \\
NT-proBNP, pg/mL & $576 \pm 414$ & $543 \pm 644$ & 0.790 \\
Cr, mg/dL & $0.81 \pm 0.18$ & $0.80 \pm 0.19$ & 0.778 \\
Echocardiography & & & \\
LVEF, \% & $54 \pm 9$ & $56 \pm 7$ & 0.283 \\
LAD, mm & $41 \pm 6$ & $39 \pm 7$ & 0.122 \\
\hline
\end{tabular}

AAD: anti-arrhythmic drug, ACEI/ARB: angiotensin converting enzyme inhibitor/angiotensin receptor blocker, AF: atrial fibrillation, BMI: body mass index, CHF: congestive heart failure, Cr: creatinine, DM: diabetes mellitus, LVEF: left-ventricular ejection fraction, HT: hypertension, LAD: left-atrial diameter, TIA: transient ischemic attack.

Table 3. VISITAG data

\begin{tabular}{|c|c|c|c|c|}
\hline & & & FTI $(n=10)$ & TAI \\
\hline Number of VISITAG, points & Number of VISITAG, points & Number of VISITAG, points & & \\
\hline left-PV & left-PV & left-PV & $35 \pm 11$ & $31=$ \\
\hline right-PV & right-PV & right-PV & $31 \pm 10$ & 35 \\
\hline FTI & FTI & FTI & & \\
\hline Left-PV & anterior & anterior & $416 \pm 104$ & 39 \\
\hline roof & roof & roof & $468 \pm 182$ & 40 \\
\hline posterior & posterior & posterior & $410 \pm 182$ & 32 \\
\hline bottom & bottom & bottom & $379 \pm 135$ & 32 \\
\hline Right-PV & anterior & anterior & $470 \pm 149$ & 38 \\
\hline roof & roof & roof & $479 \pm 181$ & 37 \\
\hline posterior & posterior & posterior & $461 \pm 134$ & 24 \\
\hline bottom & bottom & bottom & $532 \pm 318$ & 19 \\
\hline $\mathrm{CF}, \mathrm{g}$ & $\mathrm{CF}, \mathrm{g}$ & $\mathrm{CF}, \mathrm{g}$ & & \\
\hline Left-PV & anterior & anterior & $12 \pm 4$ & \\
\hline
\end{tabular}




\begin{tabular}{|c|c|c|c|c|}
\hline & & & FTI $(n=10)$ & TAI \\
\hline roof & roof & roof & $15 \pm 6$ & $15 \pm$ \\
\hline posterior & posterior & posterior & $14 \pm 6$ & $15 \pm$ \\
\hline bottom & bottom & bottom & $13 \pm 5$ & $14 \pm$ \\
\hline Right-PV & anterior & anterior & $14 \pm 6$ & $15 \pm$ \\
\hline roof & roof & roof & $15 \pm 6$ & 15 \\
\hline posterior & posterior & posterior & $16 \pm 6$ & 14 \\
\hline bottom & bottom & bottom & $17 \pm 9$ & 12 \\
\hline RF Power, W & RF Power, W & RF Power, W & & \\
\hline Left-PV & Left-PV & anterior & $33 \pm 3$ & $34-$ \\
\hline roof & roof & roof & $32 \pm 3$ & 34 \\
\hline posterior & posterior & posterior & $26 \pm 6$ & 25 \\
\hline bottom & bottom & bottom & $27 \pm 6$ & 28 \\
\hline Right-PV & Right-PV & anterior & $31 \pm 3$ & 35 \\
\hline roof & roof & roof & $32 \pm 3$ & \\
\hline posterior & posterior & posterior & $31 \pm 2$ & \\
\hline bottom & bottom & bottom & $31 \pm 2$ & \\
\hline Impedance Drop, Ohm & Impedance Drop, Ohm & Impedance Drop, Ohm & & \\
\hline Left-PV & Anterior & Anterior & $9 \pm 4$ & 10 \\
\hline roof & roof & roof & $9 \pm 5$ & \\
\hline posterior & posterior & posterior & $8 \pm 4$ & \\
\hline bottom & bottom & bottom & $9 \pm 5$ & \\
\hline Right-PV & anterior & anterior & $10 \pm 4$ & \\
\hline roof & roof & roof & $9 \pm 4$ & \\
\hline posterior & posterior & posterior & $8 \pm 6$ & \\
\hline bottom & bottom & bottom & $10 \pm 5$ & \\
\hline $\begin{array}{l}\text { RF Application Time, sec/point } \\
\text { Left-PV }\end{array}$ & RF Application Time, sec/point & RF Application Time, sec/point & $38+9$ & \\
\hline roof & roof & roof & $33 \pm 11$ & \\
\hline posterior & posterior & posterior & $30 \pm 11$ & \\
\hline bottom & bottom & bottom & $30 \pm 11$ & \\
\hline Right-PV & anterior & anterior & $34 \pm 8$ & \\
\hline roof & roof & roof & $34 \pm 12$ & \\
\hline posterior & posterior & posterior & $31 \pm 10$ & \\
\hline bottom & bottom & bottom & $33 \pm 10$ & $17=$ \\
\hline
\end{tabular}

AI: ablation index, $\mathrm{CF}$ : contact force, $\mathrm{PV}$ : pulmonary vein, $\mathrm{RF}$, radiofrequency.

\section{Hosted file}

WT AI Figure 120201026 semifinal.pptx available at https://authorea.com/users/309796/ articles/490257-wall-thickness-based-adjustment-of-ablation-index-improves-efficacyof-pulmonary-vein-isolation-in-atrial-fibrillation-real-time-assessment-by-intracardiacechocardiography

\section{Hosted file}

WT AI Figure 220201026 semifinal.pptx available at https://authorea.com/users/309796/ articles/490257-wall-thickness-based-adjustment-of-ablation-index-improves-efficacyof-pulmonary-vein-isolation-in-atrial-fibrillation-real-time-assessment-by-intracardiacechocardiography

\section{Hosted file}


WT AI Figure 320201026 semifinal.pptx available at https://authorea.com/users/309796/ articles/490257-wall-thickness-based-adjustment-of-ablation-index-improves-efficacyof-pulmonary-vein-isolation-in-atrial-fibrillation-real-time-assessment-by-intracardiacechocardiography

\section{Hosted file}

WT AI Figure 420201026 semifinal.pptx available at https://authorea.com/users/309796/ articles/490257-wall-thickness-based-adjustment-of-ablation-index-improves-efficacyof-pulmonary-vein-isolation-in-atrial-fibrillation-real-time-assessment-by-intracardiacechocardiography

\section{Hosted file}

WT AI Figure 520201101 semifinal.pptx available at https://authorea.com/users/309796/ articles/490257-wall-thickness-based-adjustment-of-ablation-index-improves-efficacyof-pulmonary-vein-isolation-in-atrial-fibrillation-real-time-assessment-by-intracardiacechocardiography

\section{Hosted file}

WT AI Figure 620201026 semifinal.pptx available at https://authorea.com/users/309796/ articles/490257-wall-thickness-based-adjustment-of-ablation-index-improves-efficacyof-pulmonary-vein-isolation-in-atrial-fibrillation-real-time-assessment-by-intracardiacechocardiography

\section{Hosted file}

WT AI Figure 720201026 semifinal.pptx available at https://authorea.com/users/309796/ articles/490257-wall-thickness-based-adjustment-of-ablation-index-improves-efficacyof-pulmonary-vein-isolation-in-atrial-fibrillation-real-time-assessment-by-intracardiacechocardiography 